\title{
Stem cell therapy improves heart function by triggering an acute immune response
}

$c 4$

stem cell
therapy can
improve cardiac
function after
infarct injury
in mice but
not by the
mechanisms
proposed
$>15$ years ago

Despite the dozens of clinical trials involving thousands of patients who have undergone stem cell therapy in the hopes of regenerating infarcted heart tissue and improving cardiac function, the efficacy of this approach remains questionable. Now, in a paper published in Nature, Jeffery Molkentin and colleagues report that stem cell therapy can indeed improve cardiac function but by triggering an acute inflammatory-based wound-healing response and not through the formation of new cardiomyocytes, as previously thought.

The potential of using bone marrow mononuclear cells (MNCs) that contain $\mathrm{KIT}^{+}$haematopoietic stem cells to regenerate the heart after myocardial infarction has been investigated since the early 2000 s, first in rodent models and later in patients in clinical trials. The rationale for these clinical trials is the observation that stem cell therapy imparted a modest but consistent improvement in cardiac function in animal models of myocardial ischaemic injury, but whether this improvement was associated with the generation of new cardiomyocytes was not clear. "We showed in our 2014 Nature paper that $\mathrm{KIT}^{+}$adult stem cells do not appreciably generate new cardiomyocytes in the mouse heart either at baseline, with injury or during development," explains Molkentin. "This work led us to question the premise of cardiac cell therapy in general, whether these cells ever really become cardiomyocytes when injected into the heart, and the mechanism of action underlying all human clinical trials using cell therapy."

To address these unknowns, the investigators injected strainmatched MNCs, zymosan (a potent inducer of the innate immune response) or saline into the hearts of uninjured mice. Confocal microscopy assessment of heart sections revealed histological foci of acute inflammation in MNCtreated and zymosan-treated mice but not in saline-treated mice. MNC and zymosan injections induced a robust accumulation of $\mathrm{CCR}_{2}{ }^{+}$and $\mathrm{CX}_{3} \mathrm{CR}^{+}$macrophages in the injection area, shifting the macrophage subtype content from a predominantly CCR $2{ }^{-} \mathrm{CX}_{3} \mathrm{CR} 1^{+}$ population in the naive state to a mix of CCR2 ${ }^{+}$and CCR $2{ }^{+} \mathrm{CX}_{3} \mathrm{CR} 1^{+}$cells.

Importantly, no differences in cell cycle activity at the injection site or at the distal areas of the heart were observed between MNC-injected or saline-injected mice, as assessed by immunohistochemistry. Furthermore, using a cell lineage tracing strategy, no transdifferentiation of injected MNCs or endogenous cardiac progenitor cells (CPCs) into cardiomyocytes or endothelial cells was observed.

MNCs, CPCs, zymosan or saline were also injected into the infarct border zone of mice subjected to ischaemia-reperfusion (IR) injury.
At 2 weeks after injection, mice treated with MNCs, CPCs or zymosan showed significantly improved ventricular performance and greater left ventricular end-systolic volume compared with saline-treated controls. This functional benefit was maintained for at least 8 weeks in mice injected with MNCs or zymosan. Treatment with a high dose of the immunosuppressant cyclosporine A abolished the improvement in cardiac function associated with $\mathrm{MNC}$ or zymosan injection.

Finally, injection of both viable and non-viable MNCs into the peri-infarct border zone of mice significantly reduced extracellular matrix (ECM) content, which had a positive effect on the mechanical properties of the injured heart. Isolated infarct strips from MNCinjected hearts showed a greater change in passive force generation over increasing stretch-lengthening and reduced expression of ECMassociated genes compared with saline-treated hearts after IR injury. Zymosan treatment imparted an even greater improvement in passive force dynamics than saline or MNC treatment.

To summarize, stem cell therapy can improve cardiac function after infarct injury in mice but not by the mechanisms proposed $>15$ years ago. "Adult stem cells do not regenerate the heart or make new cardiomyocytes, but injection of living cells, dead cell debris or even substances that induce inflammation all uniformly provide a minor healing benefit to the heart by inducing an acute innate immune response that augments infarction healing properties and scar dynamics," concludes Molkentin.

Karina Huynh

ORIGINAL ARTICLE Vagnozzi, R. J. et al. An acute immune response underlies the benefit of cardiac stem-cell therapy. Nature https://doi.org/10.1038/ s41586-019-1802-2 (2019) RELATED ARTICLE Menasché, P. Cell therapy trials for heart regeneration - lessons learned and future directions. Nat. Rev. Cardiol. 15, 659-671 (2018) 